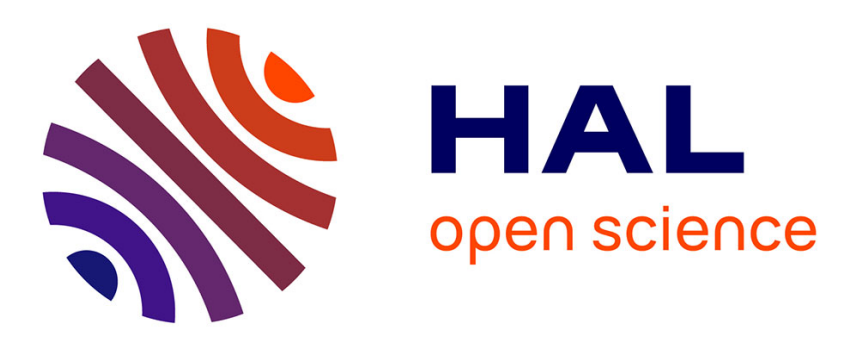

\title{
Measurement of the I-V characteristics of superconducting dipoles: automatic compensation of low frequency drift
}

E. Lesquey, F. Gire, Christophe Dolabdjian, M. Chok Sing, R. Robbes

\section{- To cite this version:}

E. Lesquey, F. Gire, Christophe Dolabdjian, M. Chok Sing, R. Robbes. Measurement of the I-V characteristics of superconducting dipoles: automatic compensation of low frequency drift. Journal de Physique IV Proceedings, 1994, 04 (C6), pp.C6-199-C6-204. 10.1051/jp4:1994632 jpa-00253127

HAL Id: jpa-00253127

https://hal.science/jpa-00253127

Submitted on 1 Jan 1994

HAL is a multi-disciplinary open access archive for the deposit and dissemination of scientific research documents, whether they are published or not. The documents may come from teaching and research institutions in France or abroad, or from public or private research centers.
L'archive ouverte pluridisciplinaire HAL, est destinée au dépôt et à la diffusion de documents scientifiques de niveau recherche, publiés ou non, émanant des établissements d'enseignement et de recherche français ou étrangers, des laboratoires publics ou privés. 


\title{
Measurement of the I-V characteristics of superconducting dipoles: automatic compensation of low frequency drift
}

\author{
E. Lesquey, F. Gire, C. Dolabdjian, M.L.Chok Sing and R. Robbes \\ Laboratoire d'Electronique et d'Instrumentation, I.S.M.RA., 6 Bd. Maréchal Juin, 14050 Caen cedex, \\ France
}

\begin{abstract}
Superconducting microbridges and Josephson junctions showing RSJ-like I-V characteristics have potential applications through their current I dependences, at fixed bias voltage $V$, versus various parameters such as temperature $(T)$, magnetic field $(B)$ or incident optical power $(\mathrm{P}) \ldots$ The main problem associated with both low values of voltage $(\mu \mathrm{V}-\mathrm{mV}$ range) and dynamic resistance $\mathrm{Rd}(0,1-10 \Omega$ range) was solved earlier ; however, the measurement system still suffers from excessive $1 / \mathrm{f}$ noise due to static biasing conditions if neither the incoming signals nor the preamplifier can be chopped. We have overcome this difficulty through the use of a periodic sampling of four points of the I-V characteristic taking advantage of the odd symmetry of this characteristic. In each period, two of the samples occur in the superconducting state and give the zero voltage reference of the measurement system. This allows us to automatically compensate for the low frequency drifts occuring at preamplifier level. The two other periodic samples, opposite in sign, must appear symmetric with respect to the zero voltage reference at the preamplifier output. This constraint is also used to automatically compensate for the low frequency drifts of the ac square signal which controls the I-V operating point. For example, a high Tc superconducting microbridge, used as a temperature sensor, has an equivalent low frequency drift of $0.4 \mathrm{mK}_{\mathrm{pp}}$ and $6 \mathrm{mK}_{\mathrm{pp}}$ respectively with and without the automatic control.
\end{abstract}

\section{INTRODUCTION}

The current biasing technic is most commonly used for superconductive dipoles because of possible operation in either the superconducting state or at low voltage values with a low resistance source. In the latter case, commercially available voltage regulation are unpracticable for the above constraints plus that of very low noise which is generally required. We worked out earlier [1] a low voltage, low noise regulation process which was found very useful to get data such as critical current Ic dependences of microbridges, Josephson junctions and dc SQUIDs, versus temperature (T), magnetic field (B) [2][3] or applied optical power (P) [4]. In the latter case, bolometric detection of far infrared radiation is a primary application, which must involve a very good stability of the bolometer assembly. Simple YBCO microbridges were proved [5] to have a high temperature coefficient :

$$
\alpha=\frac{1}{I c} \cdot \frac{d I c}{d T} \approx-0.25 K^{-1}
$$


near $80 \mathrm{~K}$ and very low temperature fluctuations down to $10^{-8} \mathrm{~K} / \sqrt{\mathrm{Hz}}$. They are good candidates as cryogenic temperature sensors to carry out the needed low drift temperature regulation, but the previous detecting scheme is limited by $1 / \mathrm{f}$ low frequency noise; of course the temperature to be regulated cannot be chopped. We describe in section 2 , a new signal processing system which overcomes this difficulty. In section 3 , we give experimental results associated to this work, and then conclude.

\section{WORKING PRINCIPLES}

Since we improved the basic circuit of [1], we start by recalling its basic operation, in order to understand the shape of time diagrams given later. Figure 1 give the notations we will use here after.

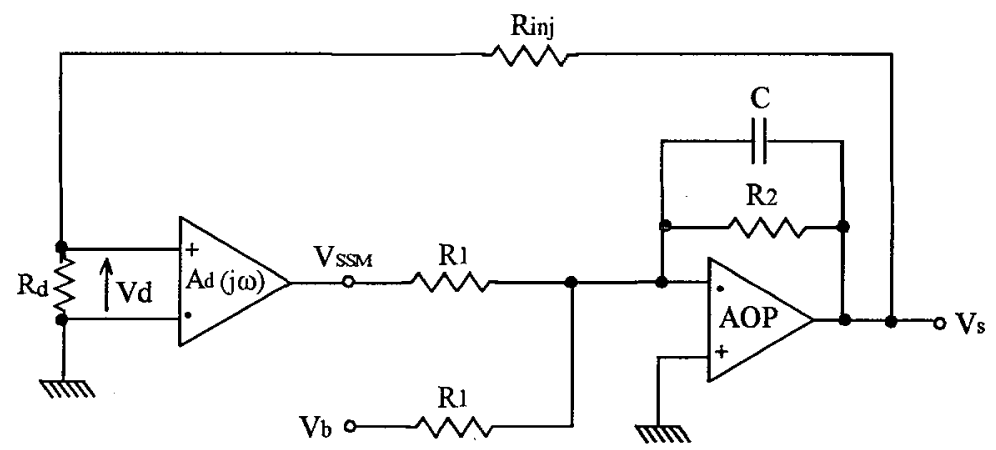

Figure 1 : Basic electronic low noise voltage regulation, $V_{s s m}$ is locked to the voltage reference $V_{b}$ and $V_{d}=V_{s s m} / A_{d}(j \omega)$.

The first stage of the circuit is made with a commercial integrated low noise instrumentation amplifier (SSM 2017) with the following specifications :

- low frequency gain : $A_{d}=1000$

- cut off frequency : $f_{d}=\omega_{d} / 2 \pi=200 \mathrm{kHz}$

- noise : en $=0.8 \mathrm{nV} / \sqrt{\mathrm{Hz}}, i_{n}=1.5 \mathrm{pA} / \sqrt{\mathrm{Hz}}$, l/f corner $\approx 100 \mathrm{~Hz}$

- input impedance : $1 \mathrm{M} \Omega$

The second stage is built around an operational amplifier (OP27) which can be considered as ideal. In the following calculations, the device under test (DUT) is a resistor $R d<<R i n j$ and we call $r$ the ratio $R_{d}\left(R_{i n j}\right.$ In the locked configuration, a straightforward calculation, in the limit $\left(\frac{R_{1}}{R_{2} A_{d}}\right)<<r<<1$ and $R_{2} C \omega_{0} \gg 1$, leads to the following expression of the voltage $V_{s s m}$ at the output of the amplifier :

$$
V_{s s m}=\frac{-V_{b}}{1-\left(\frac{\omega^{2}}{\omega_{0}^{2}}\right)+2 j \varsigma\left(\frac{\omega}{\omega_{0}}\right)}
$$

where $V_{b}$ is a reference voltage, $\omega_{0}$ is the characteristic angular velocity of the loop given by : 


$$
\omega_{0}=\left(\frac{\omega_{d}}{R_{1} C} r A_{d}\right)^{1 / 2}
$$

and $\zeta$ is the usual damping coefficient expressed as :

$$
\zeta=\frac{1}{2} \sqrt{\frac{C R_{1} \omega_{d}}{r A_{d}}}
$$

Given $A_{d}$ and $R_{I}$, the loop dynamics is governed by the two parameters $C$ and $r$; this leads to a better control facility of the loop stability. With $\zeta<1 / \sqrt{2}$, the response to a voltage step in $V_{b}$ presents oscillations around its final value ; this case should be avoided.

Let us analyse the case of superconducting dipoles with I-V characteristics symmetric with respect to the origin. The equivalent electrical circuit may be thought as a current generator with its driving current given by :

$$
I(X)=\operatorname{Sign}\left(V_{d}\right) \cdot I_{v d}(X)
$$

where $\operatorname{Sign}\left(V_{d}\right)= \pm 1$, according to the sign of $V_{d}, X$ the external parameter to be measured (T,B,P..); $I_{v d}(X)$ is the value of the current such that the total current $I$ through the DUT with $\left|V_{d}\right|$ accross its terminals, writes :

$$
I=I_{v d}(X)+V_{d} / R_{d}
$$

$R_{d}$ is then the dynamic resistance of the DUT which is generally a non-linear function of $V_{d}$, and $I_{v d}(X)$ is often a simple translation of the critical current value $I c(X)$ along the current axis. The output signal of the circuit is the voltage :

$$
V_{s}=R_{i n j} I+V_{d} \approx R_{i n j} I
$$

For dc voltage $V_{b}$, the output $V_{s}$ will then have a mean value $R_{i n j} I$ and will fluctuate around this value. It can be shown that the contribution of the voltage noise of the preamplifier, which generally dominates, leads to output fluctuations with spectral density $V_{s n}=e_{n}(f) / r$. As usual, switching the voltage $V_{b}$ at frequencies $\geq 1 \mathrm{kHz}$, allows the preamplifier to work in its white noise region. We describe now the process that was worked out.

At this level, it may be seen that the case of superconducting dipoles will complicate the loop dynamics in two ways : first, the non-linearities of $R_{d}$ will be reflected both in $\omega_{0}$ and $\zeta$ through $r$ and second, at each change of the reference polarity, the crossing of the superconducting state will cause the system to be unlocked. Figure (2) shows some time evolutions of both the device voltage $V_{d}$ and current $I$ in response to a square symetric signal $V_{b}(t)$. Curve $C$ shows that the switching between the two voltages states $V_{d}= \pm 0.4 m V$ follows the reference with some variable delay and the small plateaus in the curve correspond to the crossing of the superconducting state. 


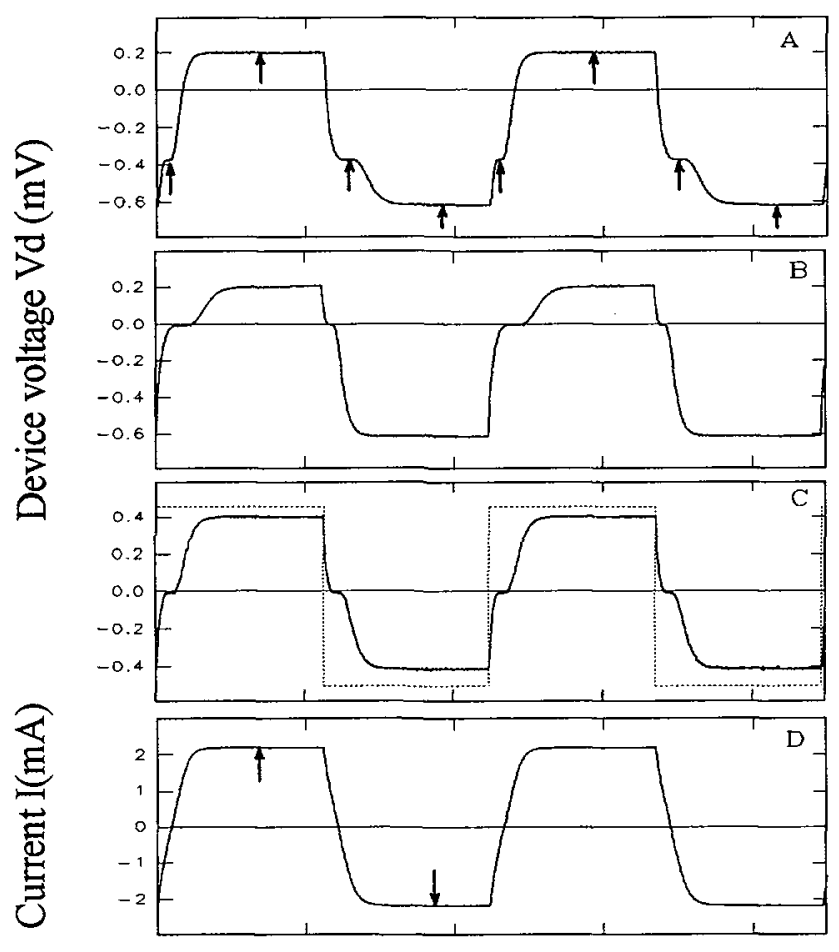

Time : 1 period $=1 \mathrm{~ms}$

Figure 2 : Time diagrams of device voltage $V_{d}(\mathrm{~A}, \mathrm{~B}, \mathrm{C})$ and current $I$ (D) in response to a square symmetric voltage $V_{b}$ which appears as the dashed line in (C).

Curve A shows some drawbacks associated to the use of the simplified circuit given in Fig.1:

(a) The low frequency drifts and the offset of the preamplifier have shifted down the plateau corresponding to the superconducting state.

(b) The I-V curve is asymmetric with respect to the superconducting state.

(c) Due to tricky dynamic aspects of the loop closed by the non linear dipole, the length of the plateau is not constant. These interesting aspects will be developped elsewhere.

The idea for compensating (a) is to sample the voltage DUT in the superconducting state which is a quasi ideal reference level. Point (c) makes difficult the exact knowledge of the delay between the step in $V_{b}$ and the beginning of the superconducting plateau whose length is related to the time constant $R_{2} C$, but not entirely determined by it. To overcome this difficulty, we use a zero crossing detector (ZCD) (see fig.3) to detect the instant when the current $I$ through the device is zero. At that instant $V_{s s m}$ is sampled and then low pass filtered (box A, fig.3). The resulting voltage allows the correction of the low frequency drifts between the DUT and the SSM output; $V_{c}$ defined on fig.3, and plotted fig.2-B clearly show the effect of that compensation. The circuit has been further improved, in order to compensate additional drifts responsible of the I-V asymmetry in fig.2-B. In that case, a zero crossing detector with a delay (ZCDD) drives the sampling of the voltage $V_{c}$; it allows after integration a correction of the driving signal $V_{b}$. The result on the final value of $V_{c}$ is plotted on fig.2-C, which 


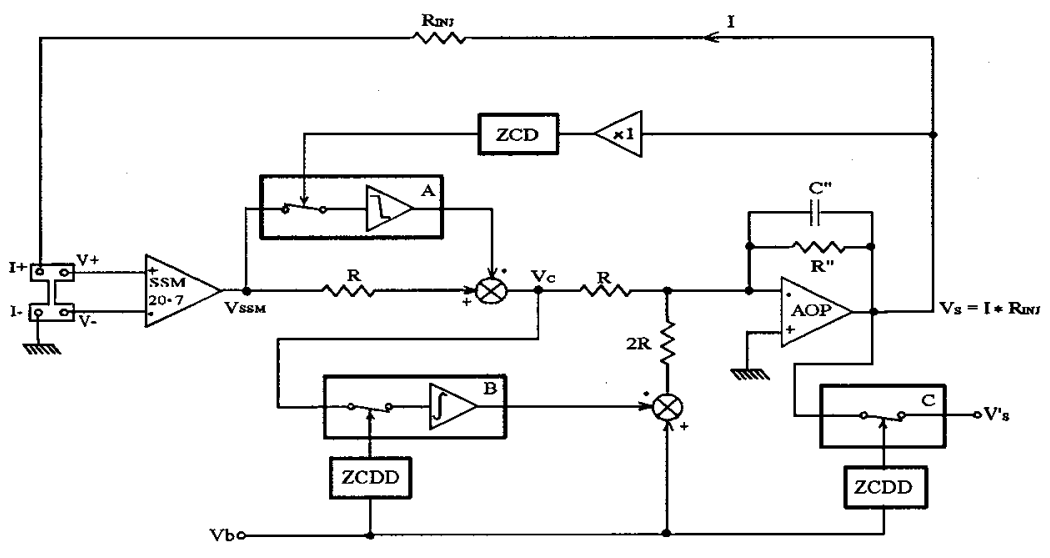

Figure 3 : Schematic of the compensating circuit explained in the text.

clearly shows the effect of the overall system fig. 2-D shows the current $I$ returned to the device ; a last sampling on the positive edge of $V_{b}$ with an additional delay then gives the output $V_{s}^{\prime}$ (see fig.3) of the system. This output reflects only the low frequency variations of the input parameter $X$ defined in (5). In our demonstrator, the switching frequency is $1 \mathrm{kHz}$, so that the upper frequency of the $\mathrm{X}$ parameter spectrum is limited to about $100 \mathrm{~Hz}$.

\section{EXPERIMENTAL RESULTS}

A YBCO/MGO microbridge was biased successively with the circuits given fig. 1 and fig.3. Fig. 4 shows the I-V characteristics of that microbridge which exhibits a large current sensitivity $S i$ of about $20 \mathrm{~mA} / \mathrm{K}$ at $1 \mathrm{mV}$ and in the vicinity of $86 \mathrm{~K}$. This microbridge can be used as a temperature sensor and the measured output fluctuations were then divided by the transfer function $\Delta V^{\prime} s / \Delta \mathrm{T}=R i n j S i$ to get the equivalent temperature fluctuations at the input. The time records reported on the Fig. 5 are those of temporal fluctuations referred to the input. Records A and B, plotted on the same scale, where taken respectively without and with our compensating technic and they clearly show the benefit associated to the use of our circuit. From fig. 5-A and 5-C, it can be seen that we improved by more than an order of magnitude the peak to peak equivalent temperature fluctuations which where reduced from $6 \mathrm{mK}$ to about $0.4 \mathrm{mK}$.

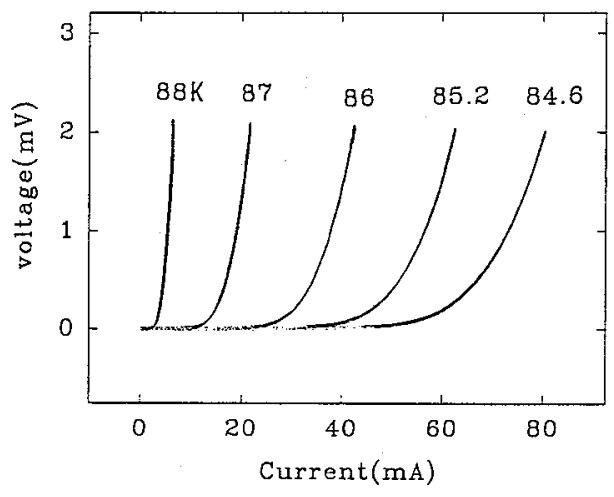

Figure 4 : I-V Characteristics of a $0.2 \times 40 \times 60 \mu \mathrm{m}^{3} \mathrm{YBCO} / \mathrm{MgO}$ microbridge at temperatures given in the inset. 


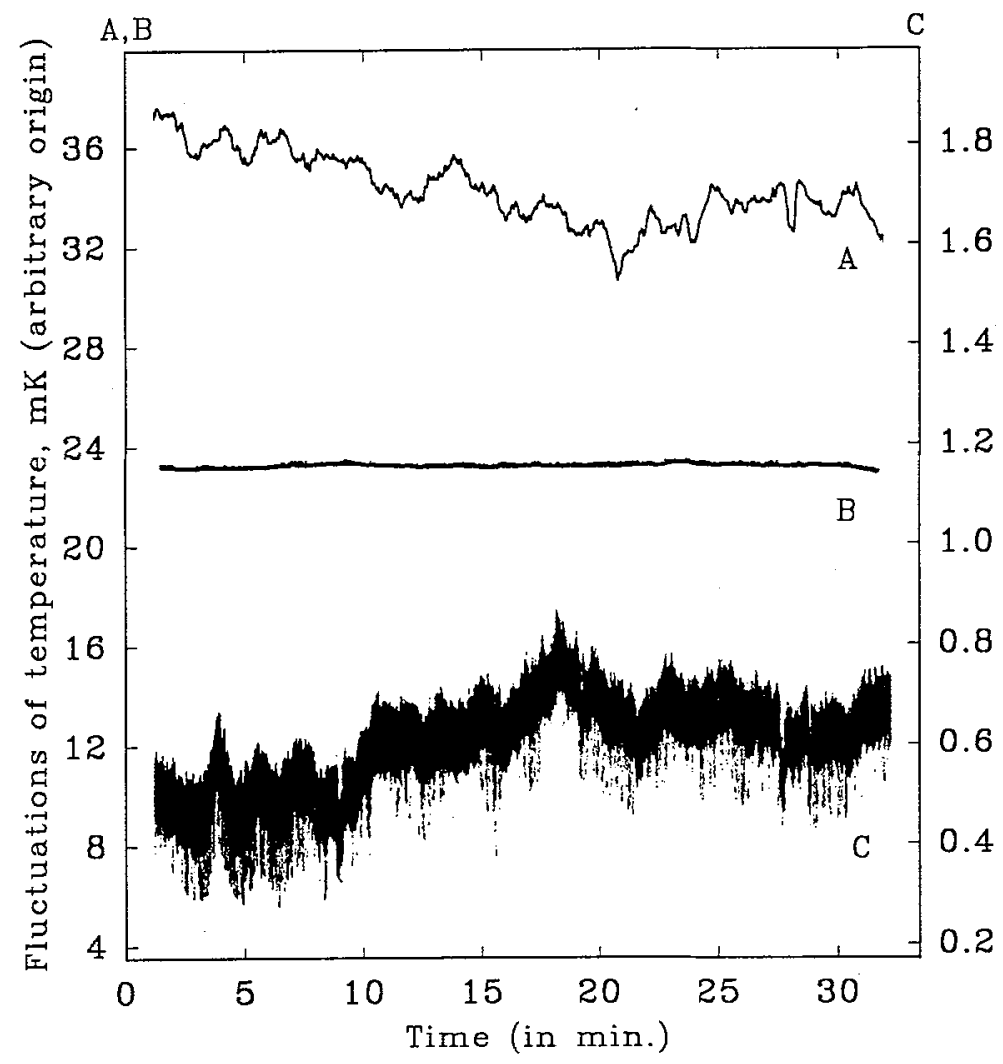

Figure 5 : Time evolutions of the equivalent temperature fluctuations referred to the input of the sensor (YBCO microbridge). Curves $\mathrm{A}$ and $\mathrm{B}$ respectively without and with our circuit, have the same scale on the left. Curve $C$ is an enhancement of B and refers to the right scale.

\section{CONCLUSION}

Our compensating technic of very low frequency drifts in the measurments of some superconducting dipoles such that microbridges, Josephson junction, de SQUIDs, was demonstrated in the case of a microbridge used as a thermometer and we lowered the low frequency noise by more than an order of magnitude. Knowing the minimum noise of such devices, sometimes down to $10^{-8} \mathrm{~K} / \sqrt{\mathrm{Hz}}$, we hope better results and presently work on an ultra low noise temperature regulation.

\section{References}

[1] Dolabdjian C., Robbes D., Lesquey E. and Monfort Y., Rev. Sci. Instrum. 642 (1993) 821-822.

[2] Dolabdjian, PhD Dissertation (Université de Caen, CAEN, 1994), pp. 59-71 and 97-100.

[3] Humphreys R.G. and Edwards J.A., Physica C 120 (1993) 42-54.

[4] Langlois, PhD Dissertation (Université de Caen, CAEN, 1993) pp. 168-185.

[5] Robbes D; Rapport d'habilitation à diriger les recherches (Université de Caen, CAEN, 1994), document $\mathrm{N}^{\circ} 22$. 